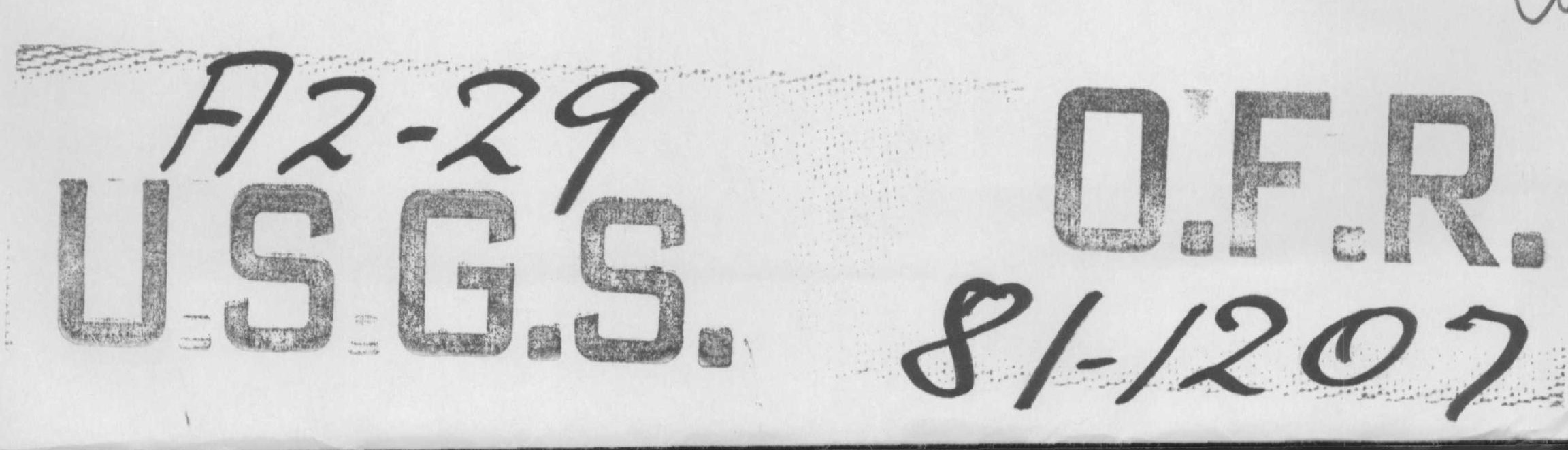


UNITED STATES DEPARTMENT OF INTERIOR GEOLOGICAL SURVEY

\section{Lithologic Descriptions, Core and Cutting Samples, Marfano Lake-Lake Valley Drilling Project, McKinley County, New Mexico, Hole Number 1}

\section{by}

A. R. Kirk, A. C. Huffman, Jr., D. J. Hammond, K. Robinson, J. Larson, D. Armstrong, and $P$. Hildebrandt

\footnotetext{
Open File Report 81-1207
}

1981

This report is preliminary and has not been reviewed for conformity with USGS editorial standards and stratigraphic nomenclature. 
Table of Contents

Page

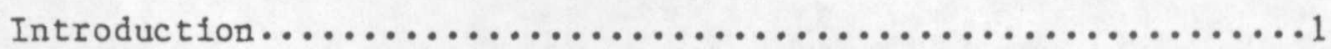

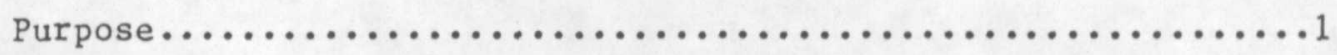

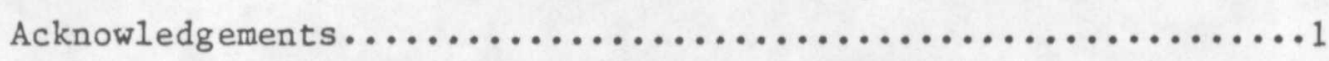

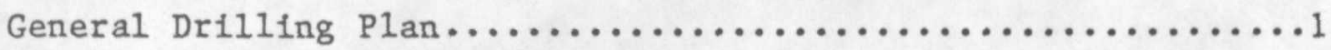

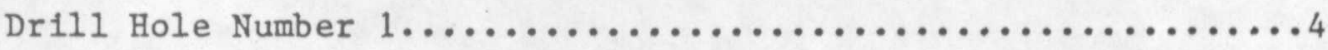

References Cited.................................

Tables

Table 1. Description of cuttings samples from Mariano

Lake-Lake Valley Drilling Profect Hole

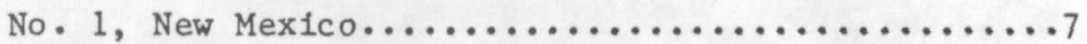

Table 2. Description of core from Mariano Lake-Lake Valley

Drilling Project, Hole No. 1, New Mexico.........12

Illustrations

Figure 1. Location of U.S.G.S. Drill Holes, Gallup

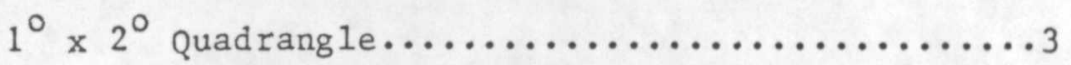

Figure 2. Location of U.S.G.S. Drill Hole no. 1,

Mariano Lake $71 / 2^{\prime}$ Quadrangle, T.16N.,

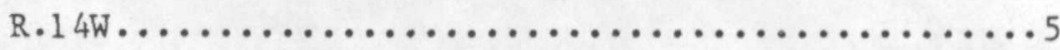




\section{INTRODUCTION}

In the fall of 1980 , the U.S. Geological Survey contracted with Longman Drilling Company of Albuquerque, New Mexico to rotary drill and core twelve holes along a north-south line from Mariano Lake to the vicinity of Lake Valley, New Mexico. This report contalns the lithologic descriptions of core and cutting samples from drill hole no. 1 .

The drilling project was funded under a reimbursable interagency agreement between the U.S. Bureau of Indian Affalrs (BIA) and the U.S. Geological Survey (USGS). The program was designed by representatives of the BIA, USGS, and the Minerals Department of the Navajo Tribe.

\section{PURPOSE}

The principal objective of this project was to provide core samples and geophysical logs for petrologic, sedimentologic, geophysical, and geochemical studies of the Upper Jurassic Morrison Formation. Other objectives included the following: stratigraphic and coal studies of Upper Cretaceous rocks; hydrologic and water monftoring of well no. 2; control for a proposed seismic study of the same geographic area; and development of water wells by the Navajo Tribal Water and Sanitation Department.

\section{ACKNOWLEDGEMENTS}

The USGS wishes to acknowledge the cooperation of Teton Exploration Drilling, Inc., and New Mexico and Arizona Land Corporation for permission to drill hole no. 1 on their mineral lease.

\section{GENERAL DRILLING PLAN}

The locations of all twelve drill holes are shown on figure 1, which is a portion of the Gallup $1^{\circ} \times 2^{\circ}$ Quadrangle. The general drilling plan called for most holes to be rotary drilled into the Upper Cretaceous Dakota Sandstone and then cored into or through the Recapture Member of the Morrison 
Formation. The interval to be cored in each hole was about $600 \mathrm{ft}$.

Exceptions to the general drilling plan were as follows: Hole no. 2, rotary drilled, surface to Jurass1c Entrada Sandstone; Hole no. 4A, cored 21$218 \mathrm{ft}$, to test an observed near surface I.P. anomaly; Hole no. 6, deepened after coring by rotary drilling into the Jurassic Entrada Sandstone; Hole no. 7A, cored only the Westwater Canyon Member of the Morrison Formation; Hole no. 8, abandoned in lower part of Westwater Canyon Member of the Morrison Formation; and Hole nos. 9 and 10, abandoned In Upper Cretaceous rocks. Chip samples were collected at $10 \mathrm{ft}$ or $20 \mathrm{ft}$ intervals throughout each hole and sludge samples collected at 20-ft intervals throughout the cored interval.

The following sulte of geophysical logs were included in the general drilling project: natural gamma, self potential, neutron-neutron porosity, resistance, resistivity, temperature, deviation, gamma-gamma density, caliper, magnetic susceptibility, gamma ray spectrometer (KUT), sonic, Induced polarization, conductivity, and high-resolution 4-arm digital dipmeter. 


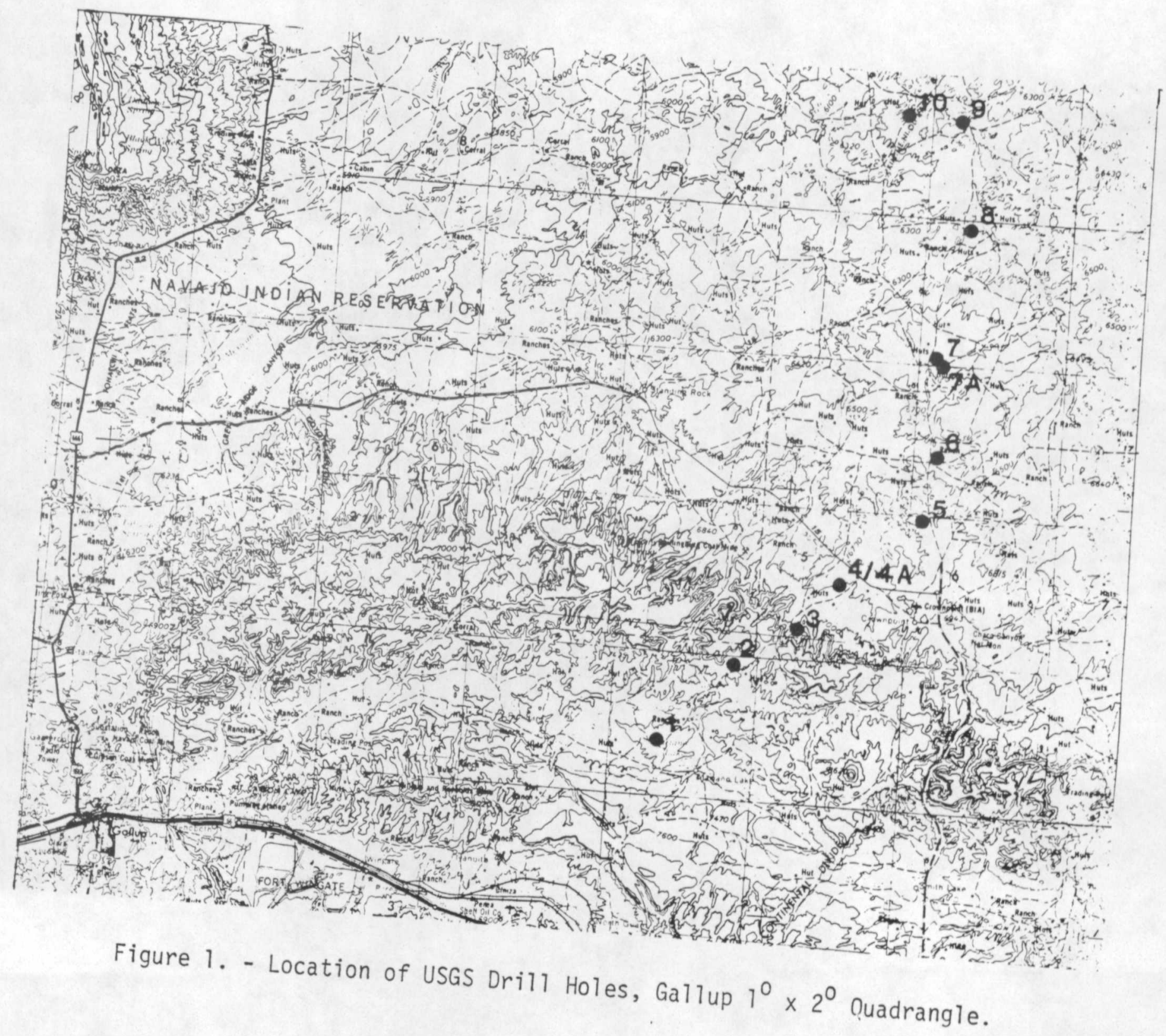




\section{DRILL HOLE NO. 1}

The location of this well is shown on figure 2 .

The vital statistics on this well include the following:

Spud date: October 21,1980

Location: T. 16 N., R. 14 W., NE/4 sec. 29 ; Lat. $35^{\circ} 35^{\prime} 30^{\prime \prime}$, Long . $108^{\circ} 22^{\prime} 12^{\prime \prime}$

Collar Elevation: $7238 \mathrm{ft}$ (topo) Mancos Shale (Cretaceous)

Core Point Top: $477 \mathrm{ft}$ (depth) Dakota Sandstone (Cretaceous)

Bottom Cored Interval: $1076 \mathrm{ft}$ (depth) Cow Springs Sandstone (Jurassic)

Total Depth: $1076 \mathrm{ft}$ (depth) Cow Springs Sandstone (Jurass1c)

Core Recovery: 70 percent

Status of we11: Abandoned, October 29, 1980.

The following suite of geophysical logs were run on this hole and have been published by the U.S. Geological Survey (1981): natural gamma, self potential, neutron-neutron porosity, resistance, resistivity, deviation, gamma-gamma density, caliper, KUT, conductivity, and magnetic susceptibility. Cutting samples from rotary drilling were collected and described on ten (10) foot intervals to the core point at $477 \mathrm{ft}$ (see Table $\#_{1}$ ). Cutting samples were collected through the cored interval 477-1076 ft but were not described. Core samples were collected in $40 \mathrm{ft}$ core runs and are $3 \mathrm{in.} \mathrm{In} \mathrm{diameter.}$ The core samples were described in the field (Table $k_{2}$ ), taped, boxed, and shipped to the USGS Core Library in Denver where they were frozen, split, photographed, and sampled (for petrography, geochemistry, heavy-mineral-sulte, clay-mineralogy, and paleomagnetic studies). A split of the core has been archived for reference and future study.

The following core and cutting sample descriptions were described in the field. The abbreviations and graphic symbols used in the core description are defined in Reynolds and others (1975). 


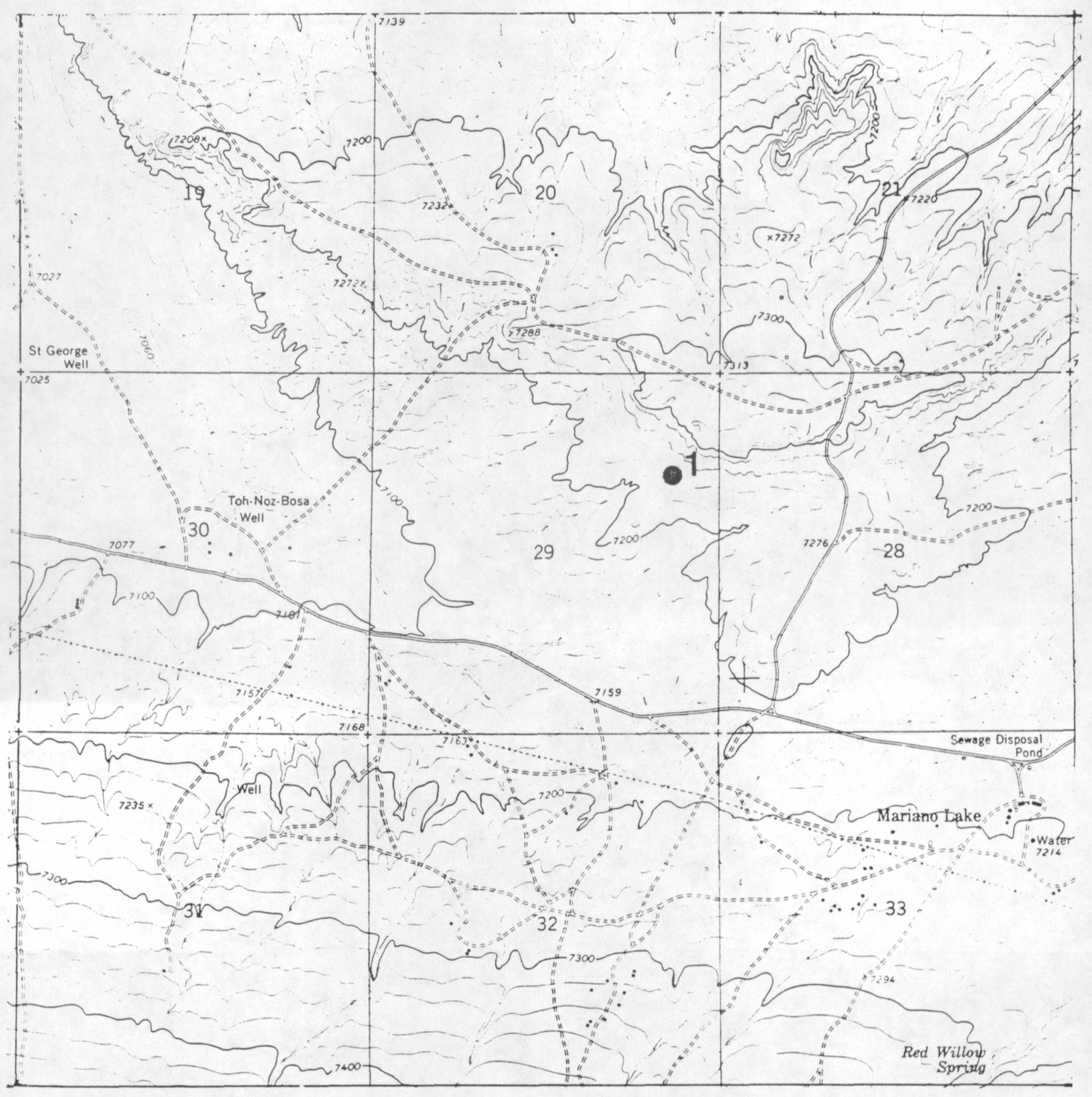

Figure 2. - Location of USGS Drill Hole 1, Mariano Lake 7 1/2' Quadrangle, T $16 \mathrm{~N}, \mathrm{R} 14 \mathrm{~W}$. 


\section{REFERENCES CITED}

Reynolds, M. W., Ahlbrandt, T. S., Fox, J. E., and Lambert, P. W., 1975, Description of selected drill cores from Paleozoic rocks, Lost Soldier 011 Field, South Central Wyoming, Part 1: U.S. Geological Survey Open-File Report $75-662,34$ p.

USGS, 1981, Geophys1cal log sulte from dr111 hole nos. 1 and 2, Marlano LakeLake Valley drilling project, McKinley County, New Mexico, USGS Open-File Report 81-172, p. 
i. hocation:

4ole No: $\frac{S-1}{5-5}$ की

है

Sec. 29 T. $16 N$ R. $14 \mathrm{~W}$ Quadrangle (9.5) MARIANO LAKE State: N.M. Date: $10 / 21 / 80$

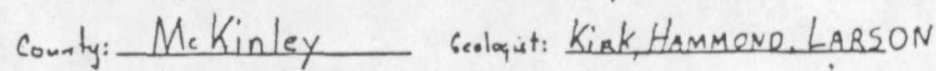

Lot/long: $\frac{35^{\circ} 35^{\prime} 30^{\prime \prime} \mathrm{N}}{108^{\circ} 22^{\prime} 12^{\prime \prime} \mathrm{W}}$. Skect 1 of 5 .

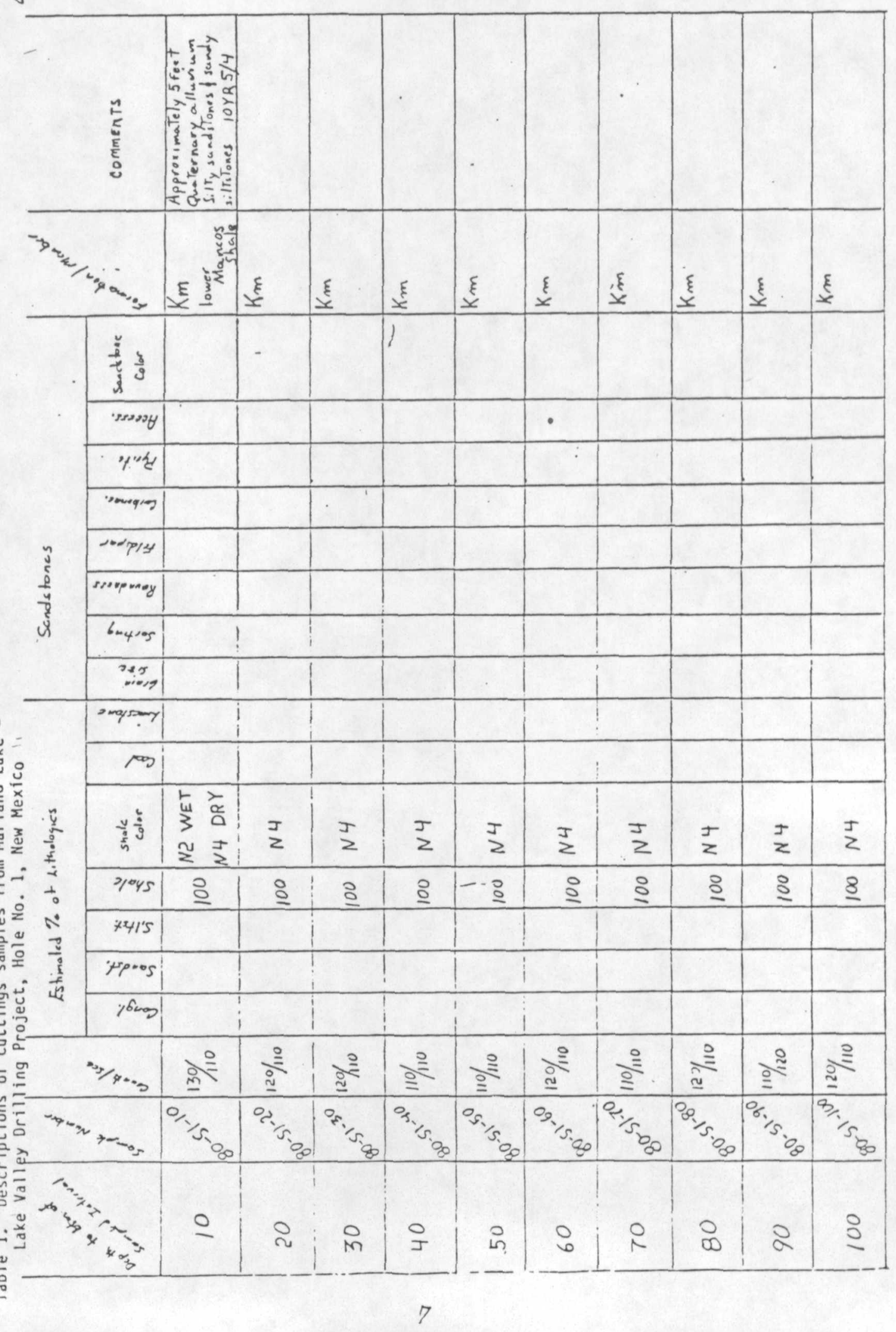


1

i. hocation:

Sec. 29 T.16N R.14W Quadro-gle (7.5) Mariane Lake

1

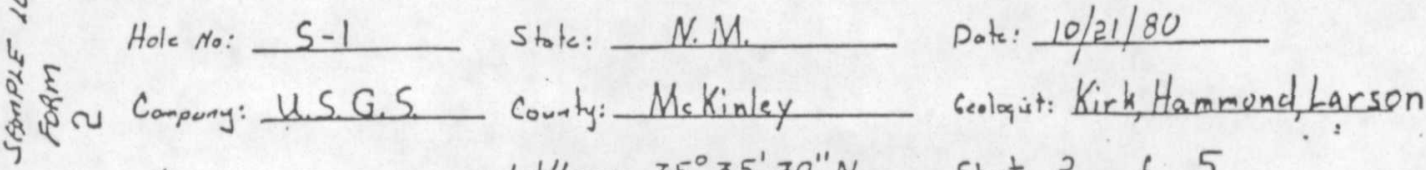

है.

Lot/loog: $\frac{35^{\circ} 35^{\prime} 30^{\prime \prime} \mathrm{N}}{108^{\circ} 22^{\prime} 12^{\prime \prime} \mathrm{W}}$. Sheet 2 of 5.

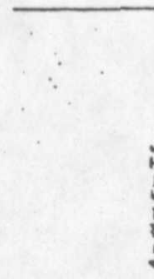

ڤ.

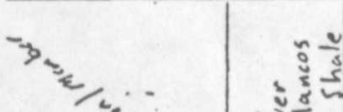

“省|

,

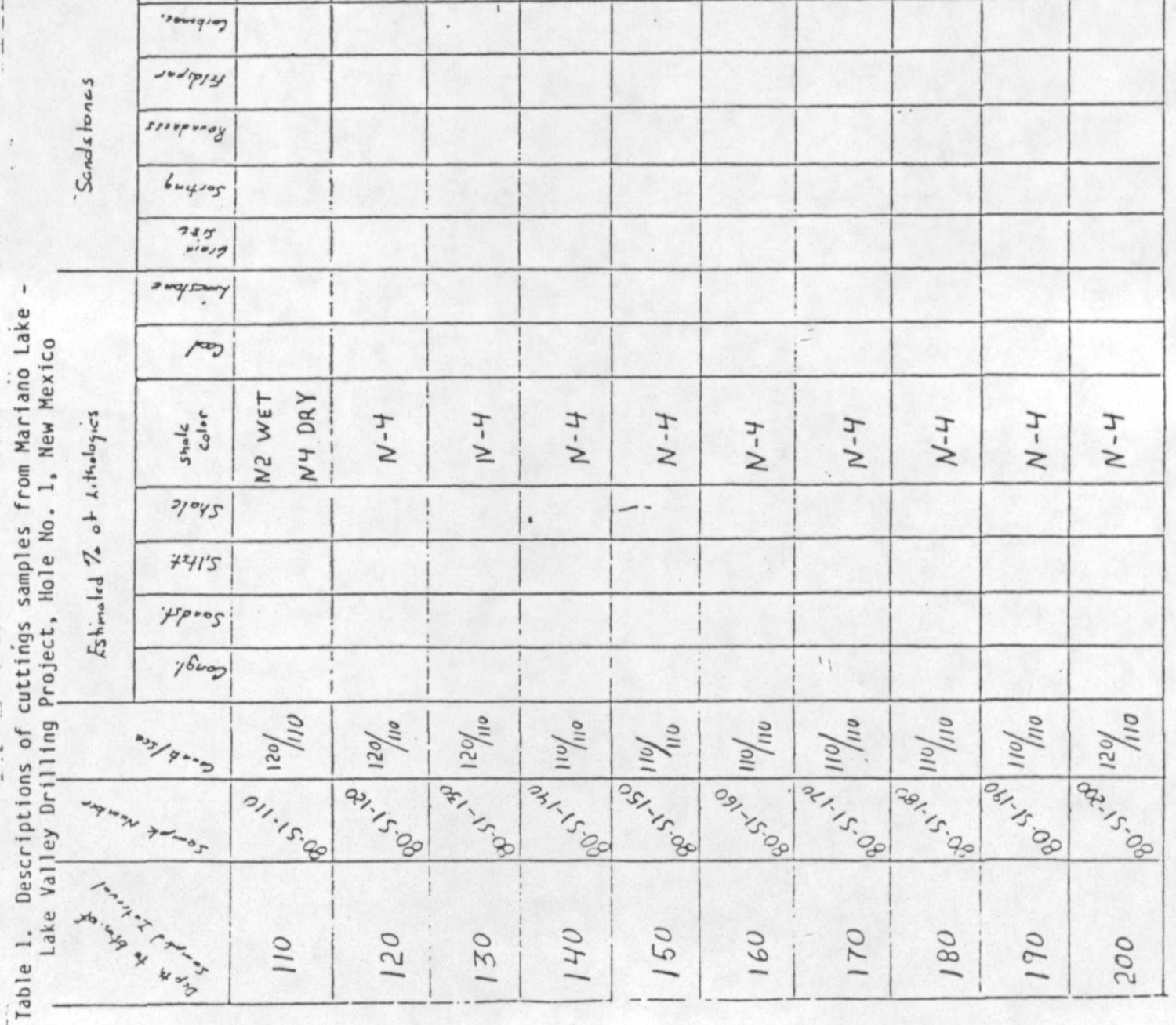

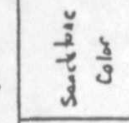
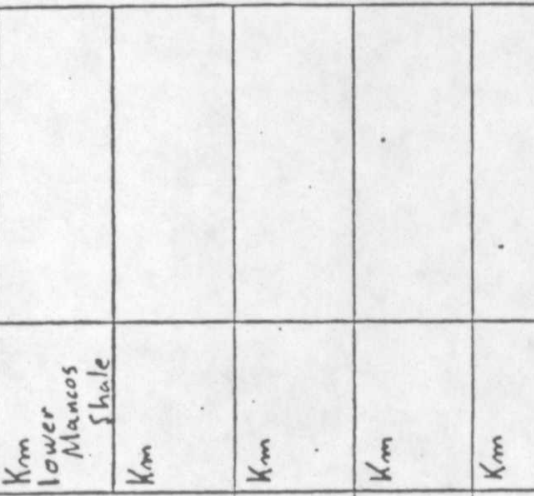

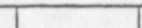
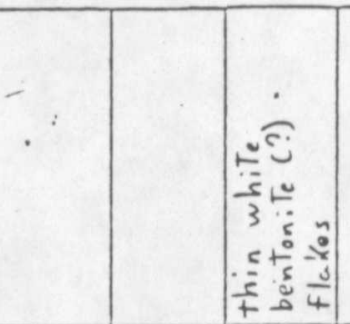
1

i. hocation:

Sec. 29 T. 16N R.14W Quadro-ģle (7.5) Mariane Lake

Hole No: $S-1$ State: N.M.

Date: $10 / 21 / 80$

OM Company: U.S.G.S. Courty: Mckinley

Gealogit: Kirk, Hammond, Larson

s.

Lot/Long: $\frac{35^{\circ} 35^{\prime} 30^{\prime \prime} \mathrm{N}}{108^{\circ} 22^{\prime} 12^{\prime \prime} \mathrm{W}}$, Sheet 3 of 5 .

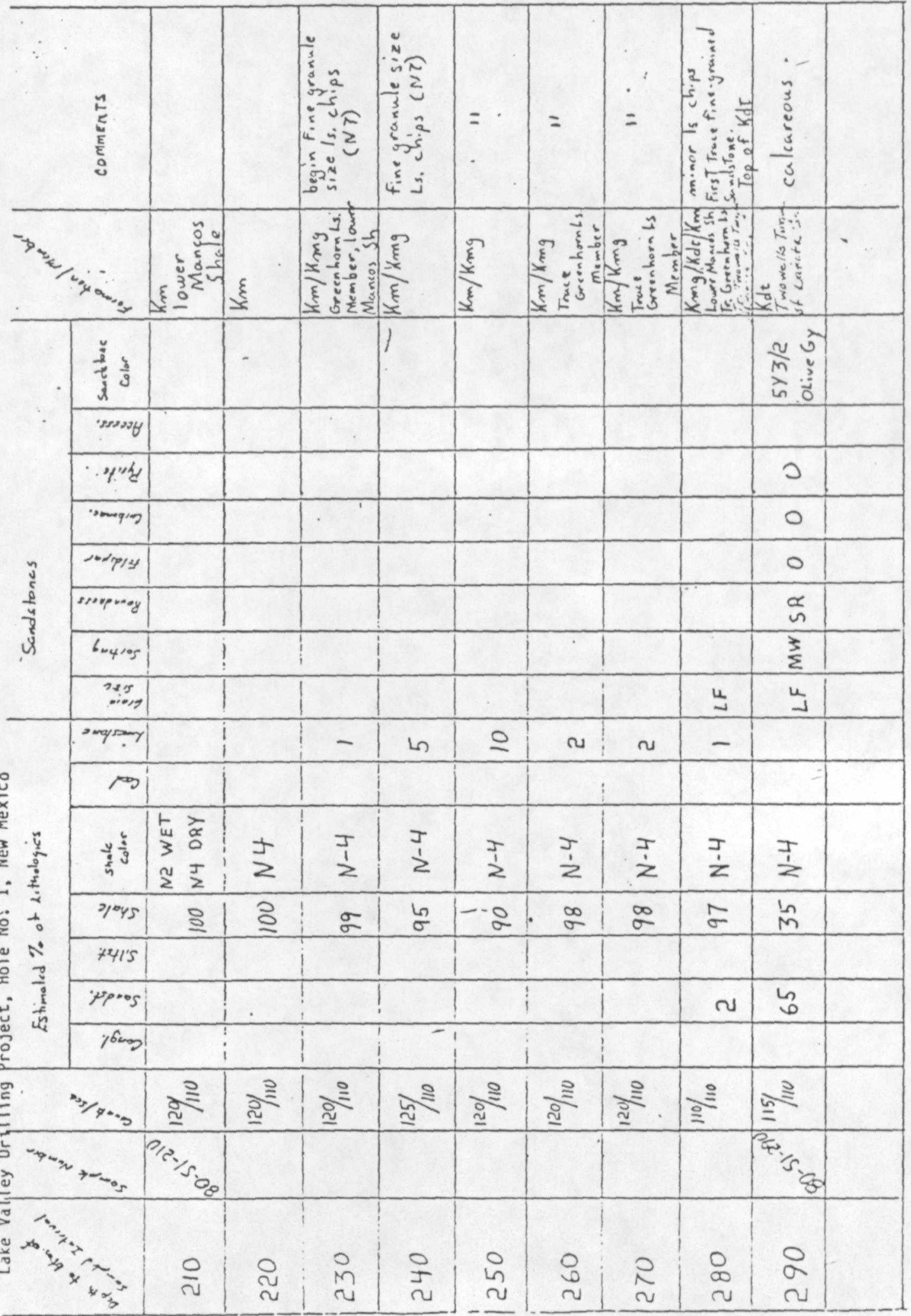


1. Socation: See. 29 T. $16 \mathrm{~N}$ R.14W Quediangle (9.5) Marianc hake i Hole No: S-1 Stote: N.M. Pote: $10 / 22 / 80$

J Compuny: U.S.G.S. County: MCKinley Geologit: HufFman, Larson s. Lot/long: $\frac{35^{\circ} 35^{\prime} 30^{\prime \prime} N}{108^{\circ} 22^{\prime} 12^{\prime \prime} W}$. Sheet 4 of 5 .

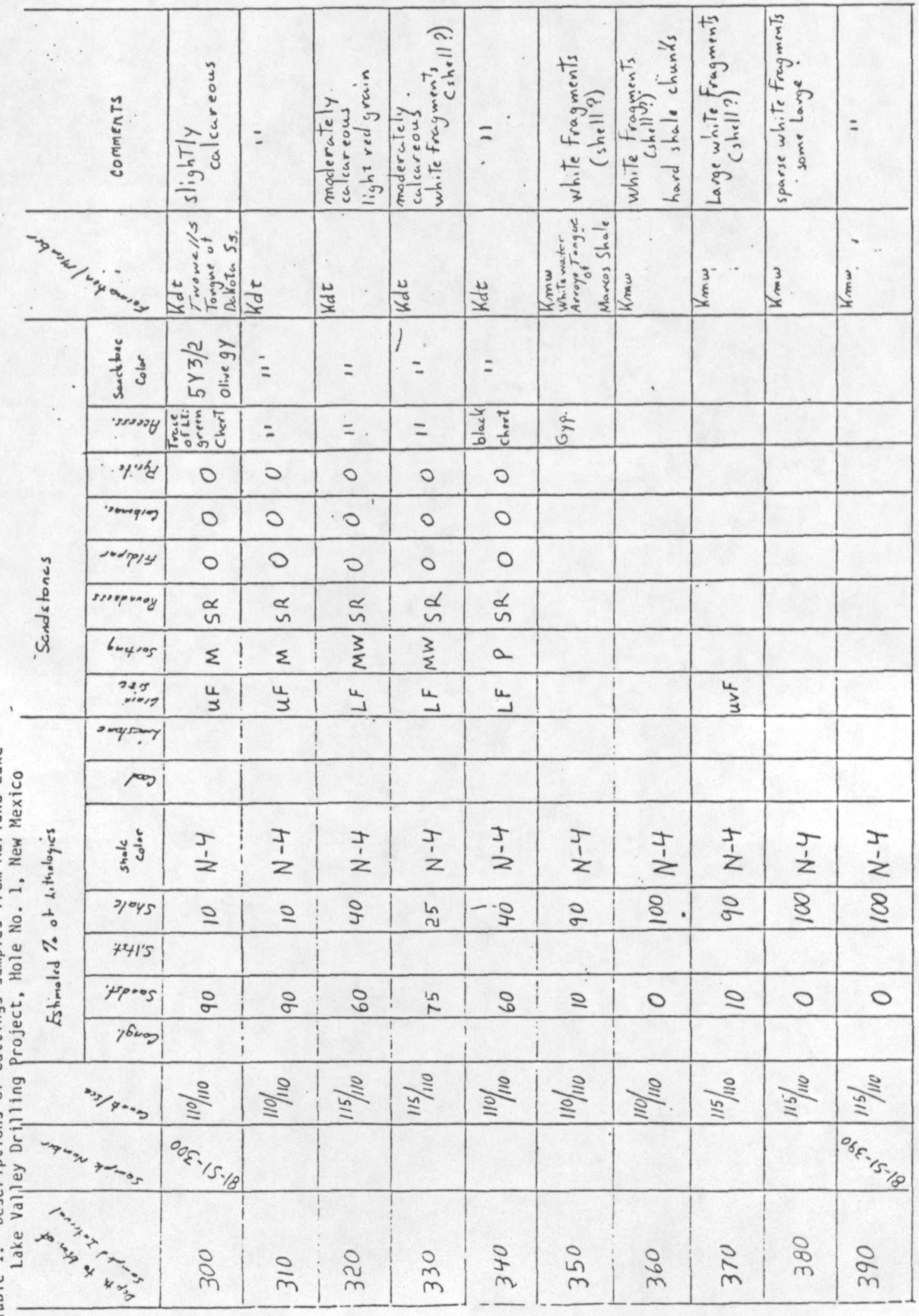


1

So Location:- Sec. 29 T. $16 \mathrm{~N}$ R. $14 \mathrm{~W}$ Quedrongle (9.5) Mariang Lake 1. Hole No: $S-1$ Stote: N.M.

$\because$ L Company: U.S.G.S. county: Mekinley Gelogit: Huff man, barsen s. Lot/long: $\frac{35^{\circ} 35^{\circ} 30^{\prime \prime} N}{108^{\circ} 22^{\prime} 12^{\prime \prime} W}$. Shect 5 of 5 .

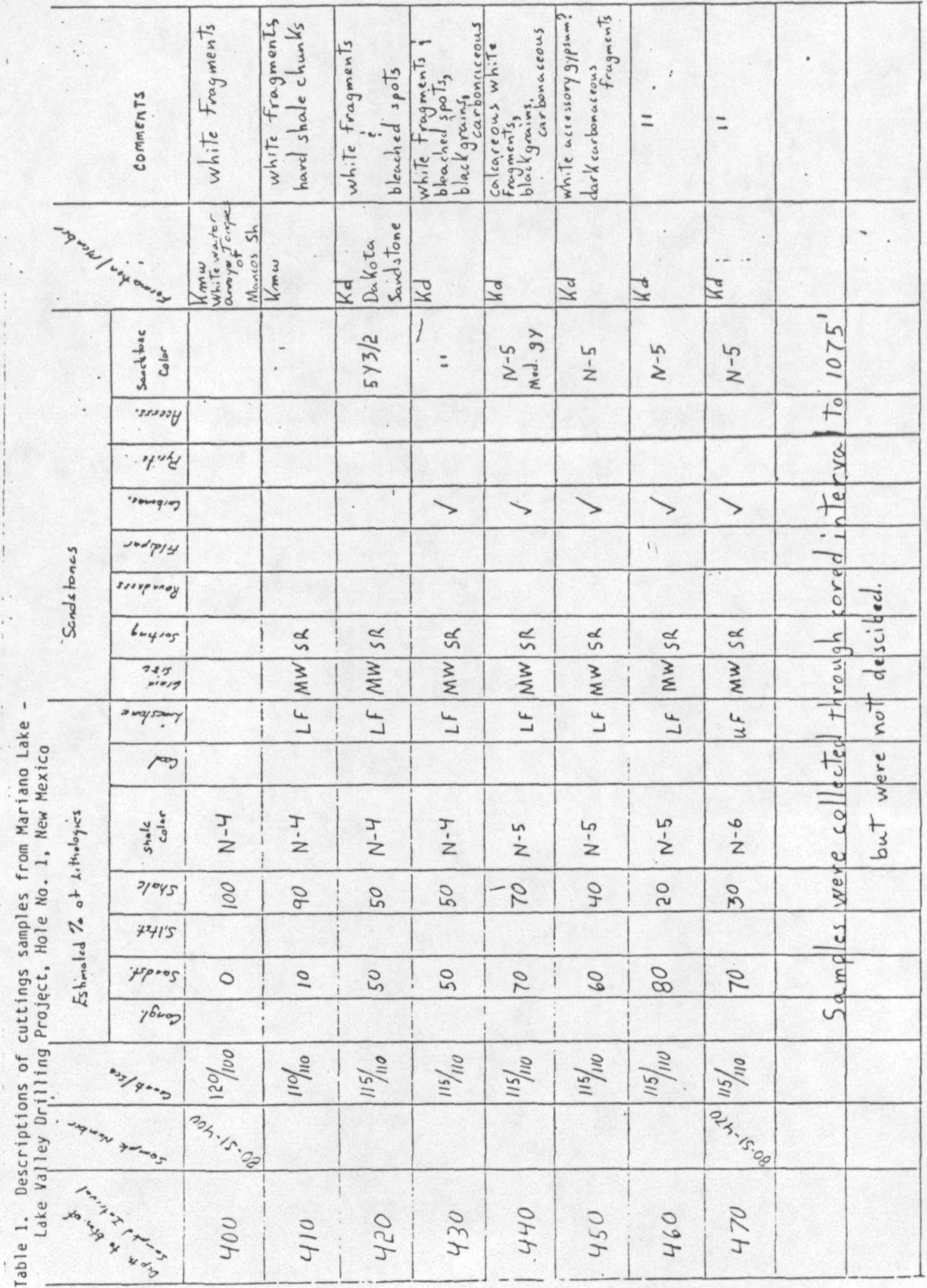


LOCATION.

STATE.

LAT.-LONG.
Sec. 2 .

COUNTY IIS ne,

$100^{\circ}=2^{\prime} 12^{\prime} \mathrm{y}$
QUADRANGTLE (7.5') Mariano boKe

DATE . 10:2?/80

GEOL. Kirk/huffmon/Hamenth/Lan

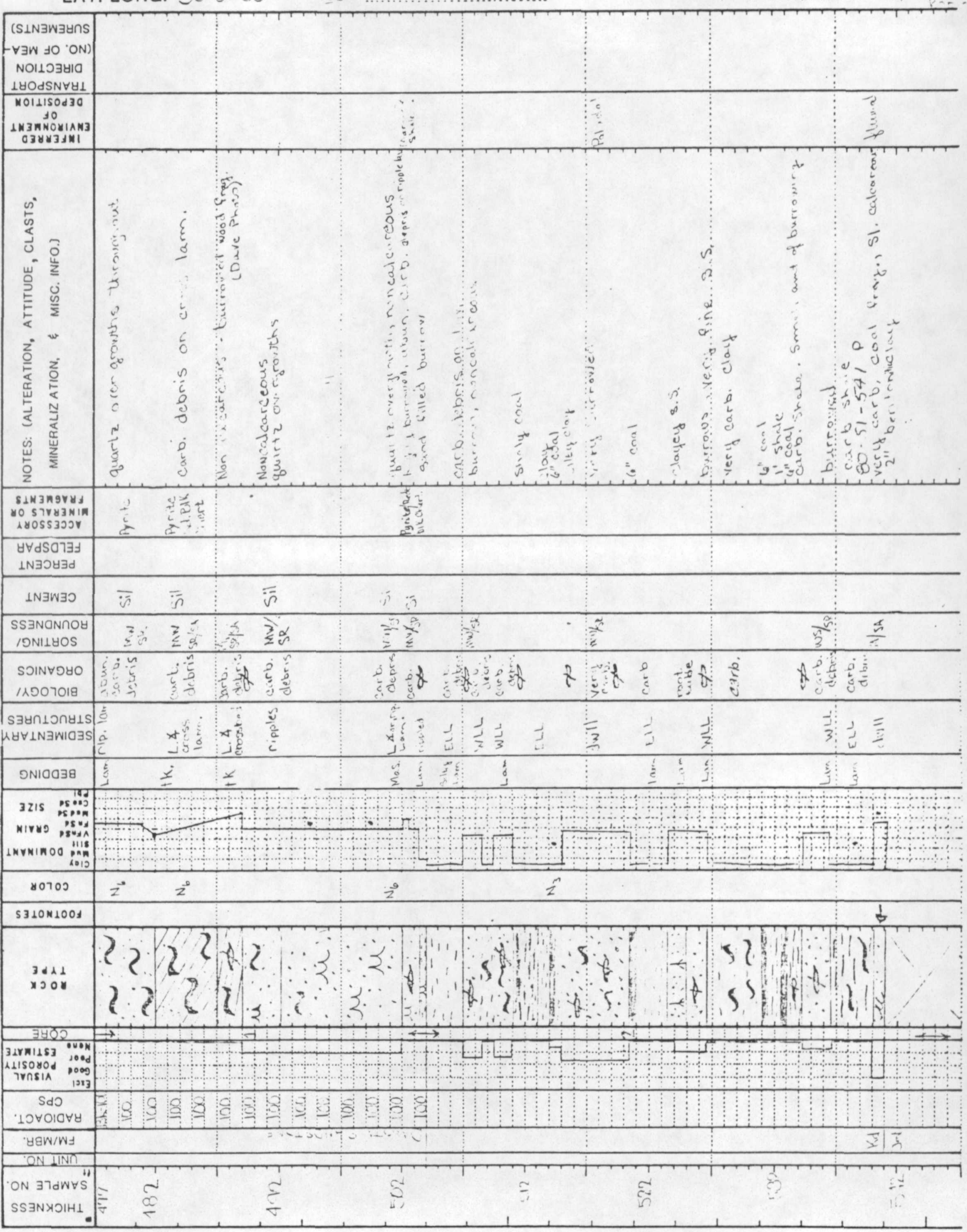




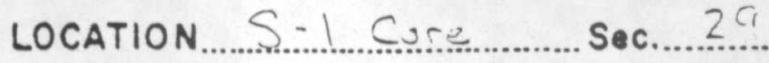

STATE

COUNTY

U.S.G.S. CORE LIBRARY NUMBER

API WELL NUMBER.

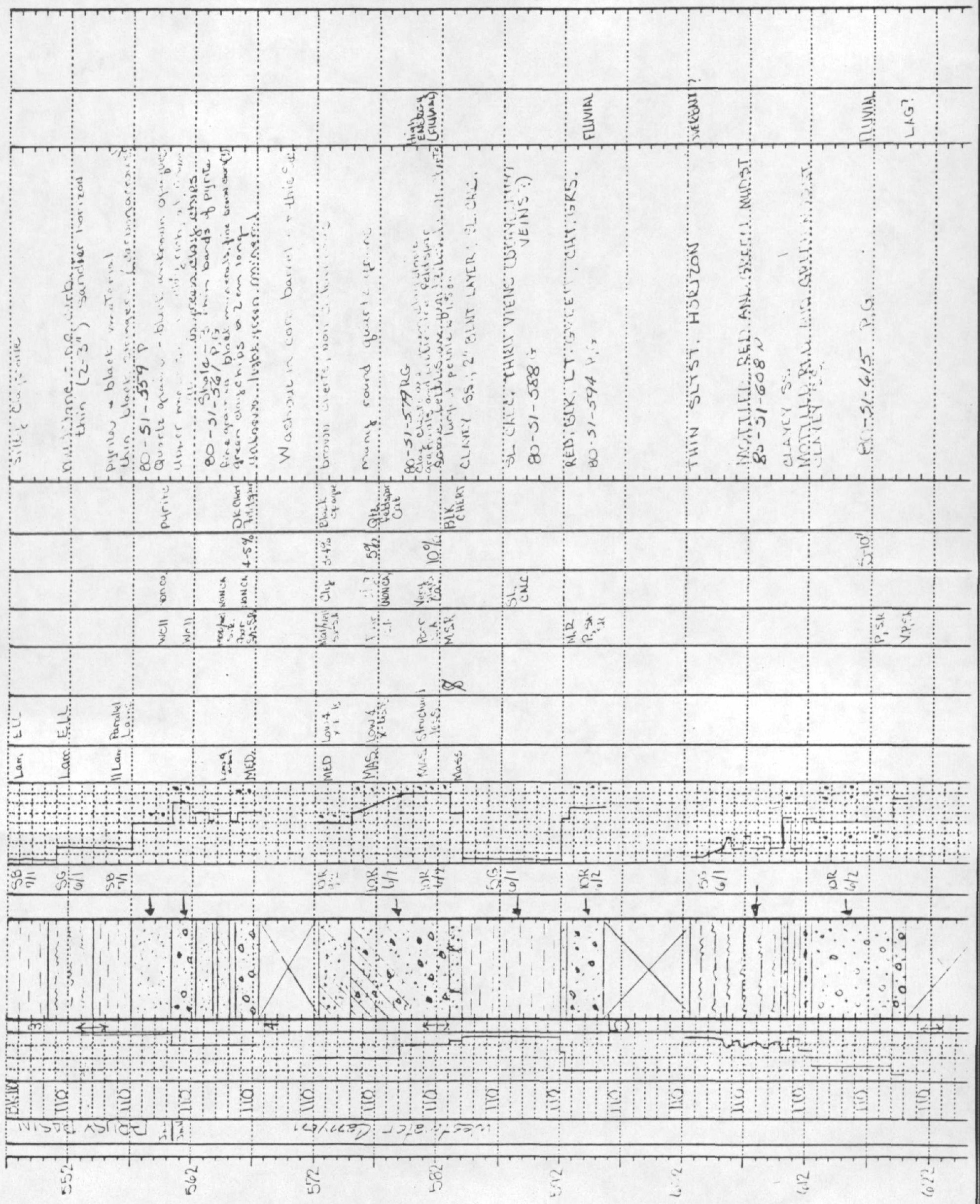




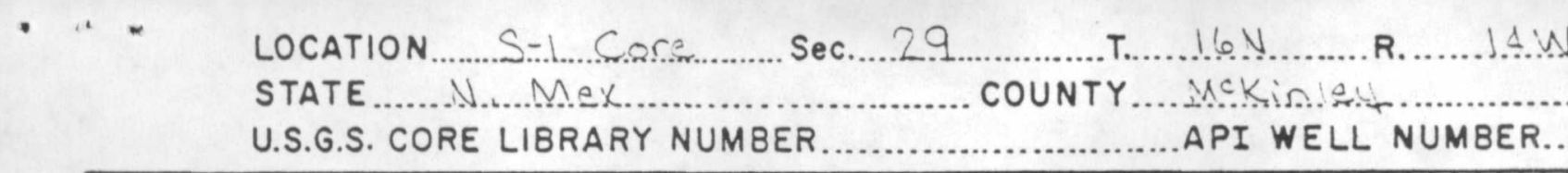

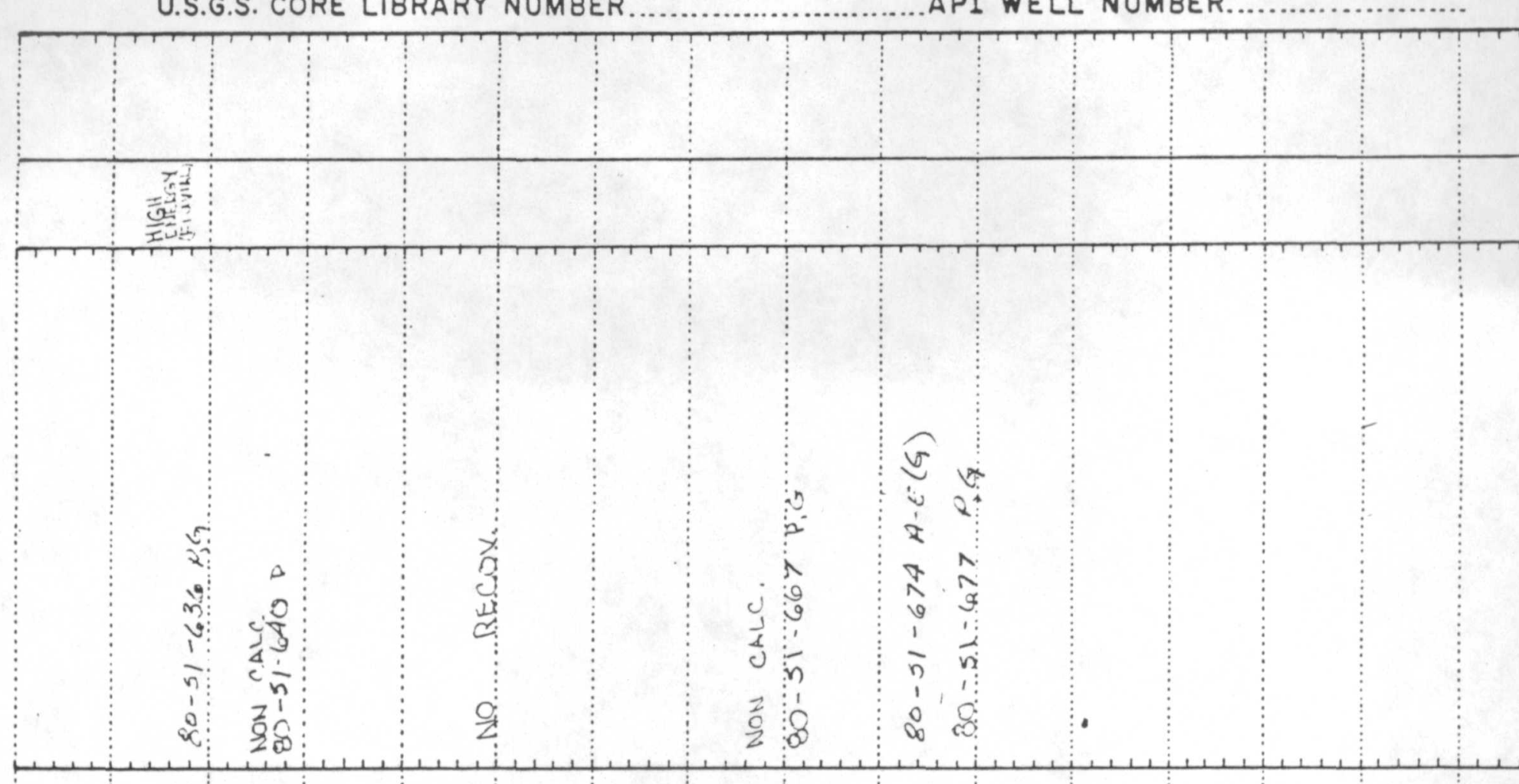

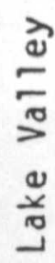

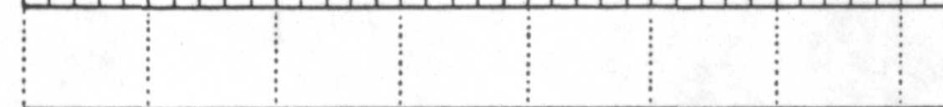

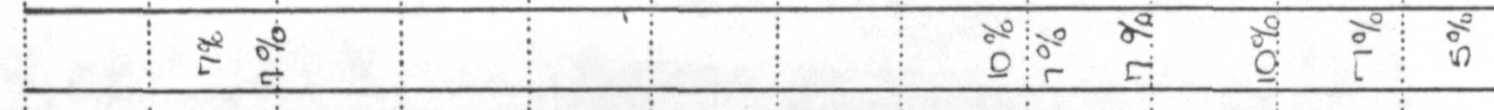

象

a $\frac{1}{x}$

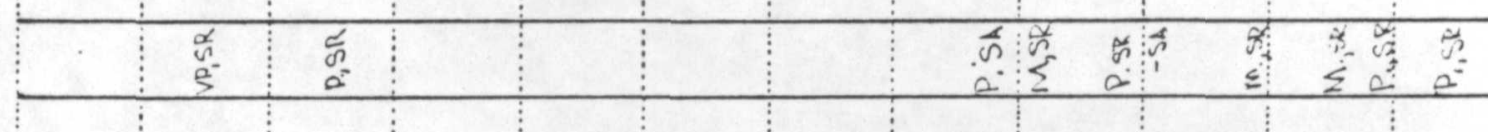

范之

을

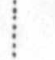

(4) +

-

인.

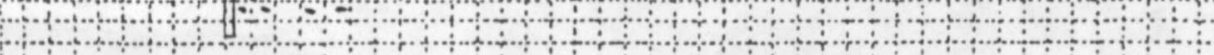

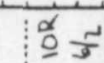

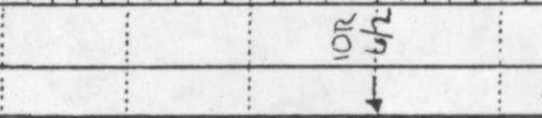

$\frac{9}{7}$

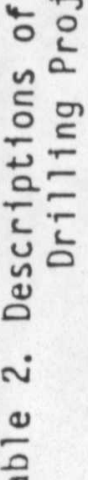

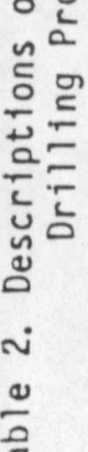
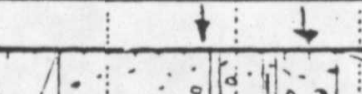

$x \rightarrow 0$

$\Delta 00.040$

L1 1.20 ol

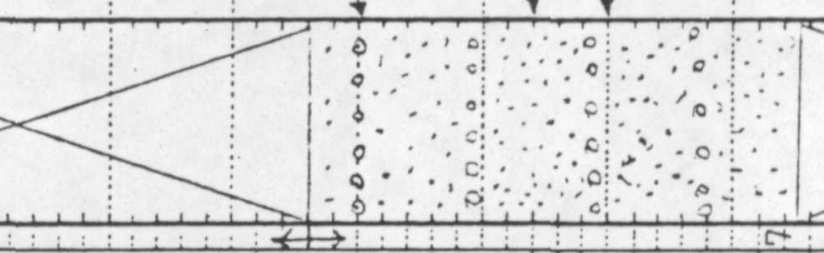

$+$

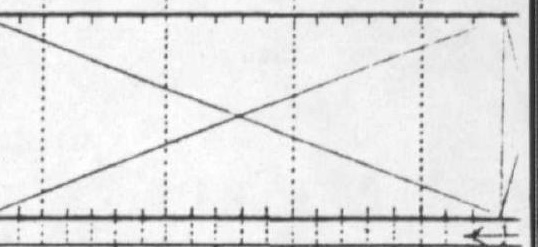

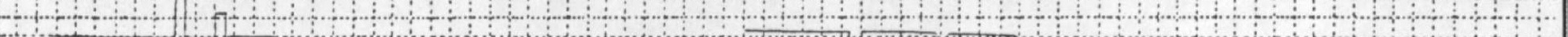

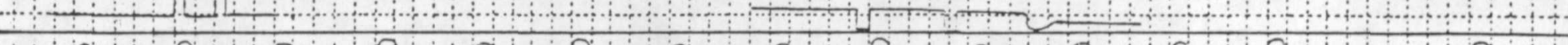

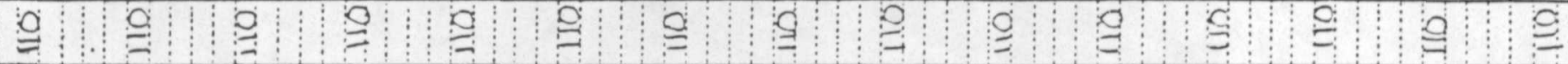

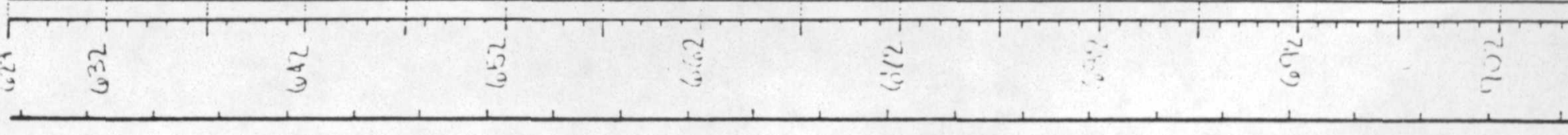


LOCATION S-I CORE HYE SeC...2.

T......16. \

R..............

STATE ....NEW ....MEX!CO

COUNTY ....WC.KINLE.

U.S.G.S. CORE LIBRARY NUMBER

API WELL NUMBER.

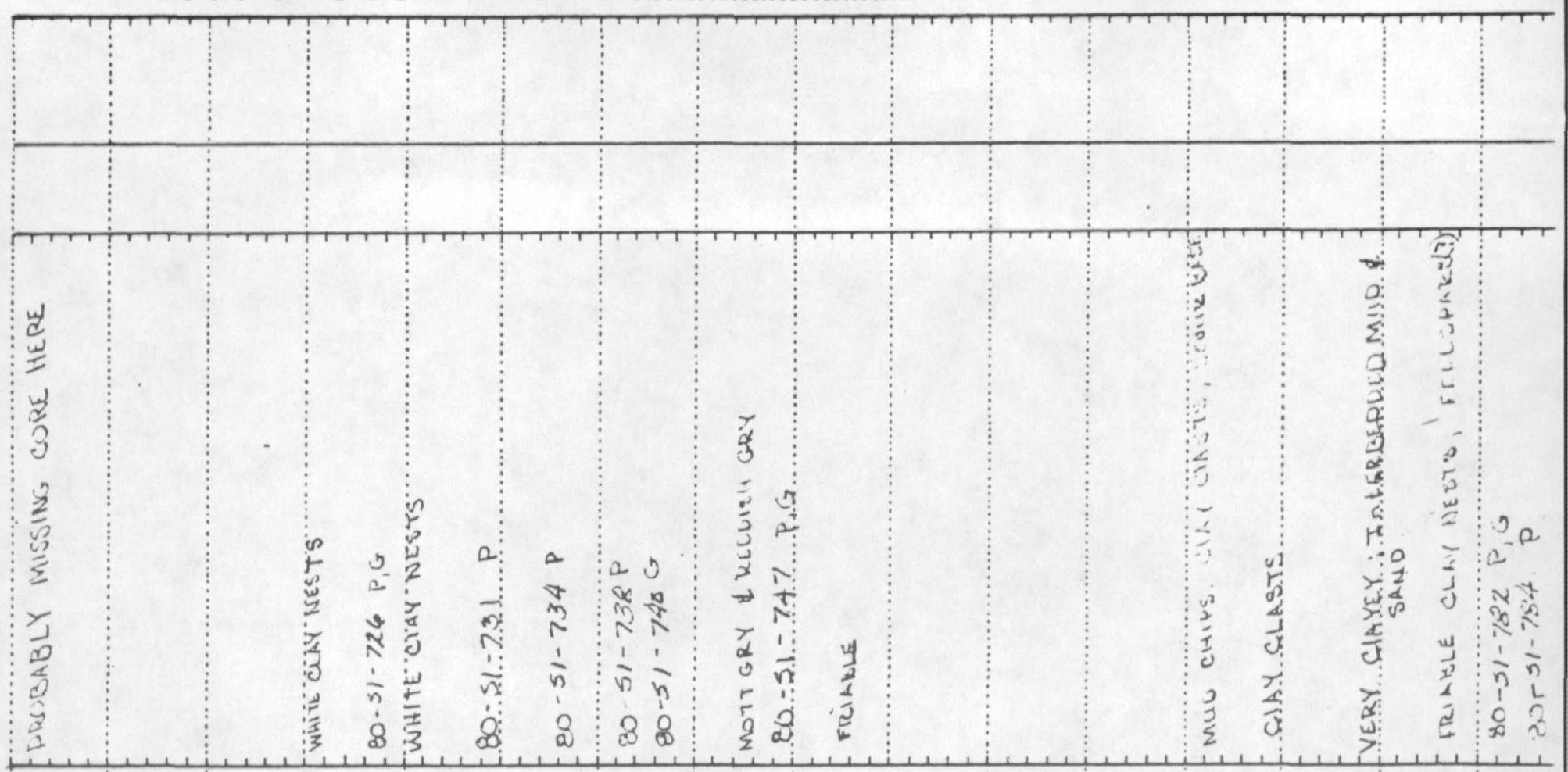

वे

व कृ

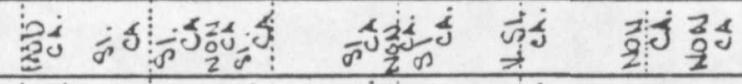

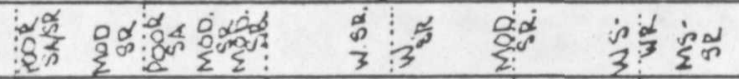

$8: 8$

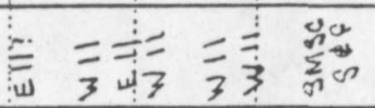

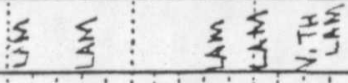

$\div \frac{1}{\check{2}}$

西

1.

$\frac{1}{2}$

읃

$\frac{1}{4}-1$

एक

-

a

눈

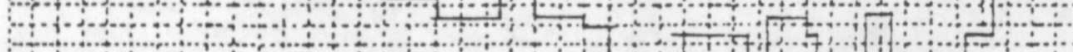

Holnd

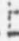

엉

$+1+\infty$

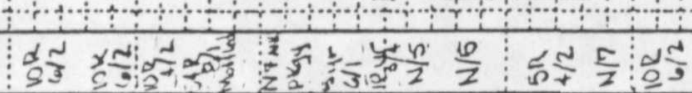

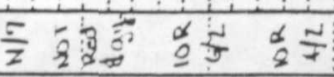

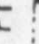

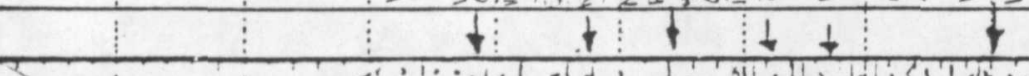

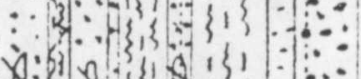

묻

:

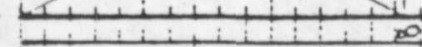

20

H $\left\{\begin{array}{l}1 \\ 1\end{array}, \therefore\right.$

$+1+1$

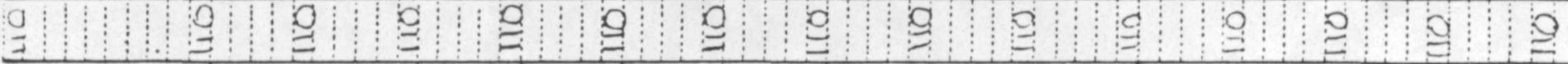

$\frac{9}{10}$

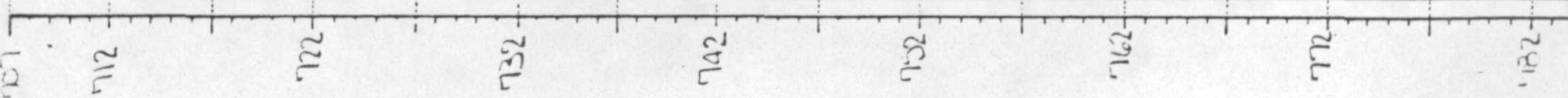




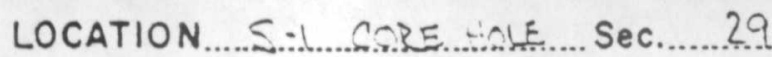

T.....16. $\mathrm{N}$.

R....t女W.

STATE ...... EW ME ME

U.S.G.S. CORE LIBRARY NUMBER

API WELL NUMBER.

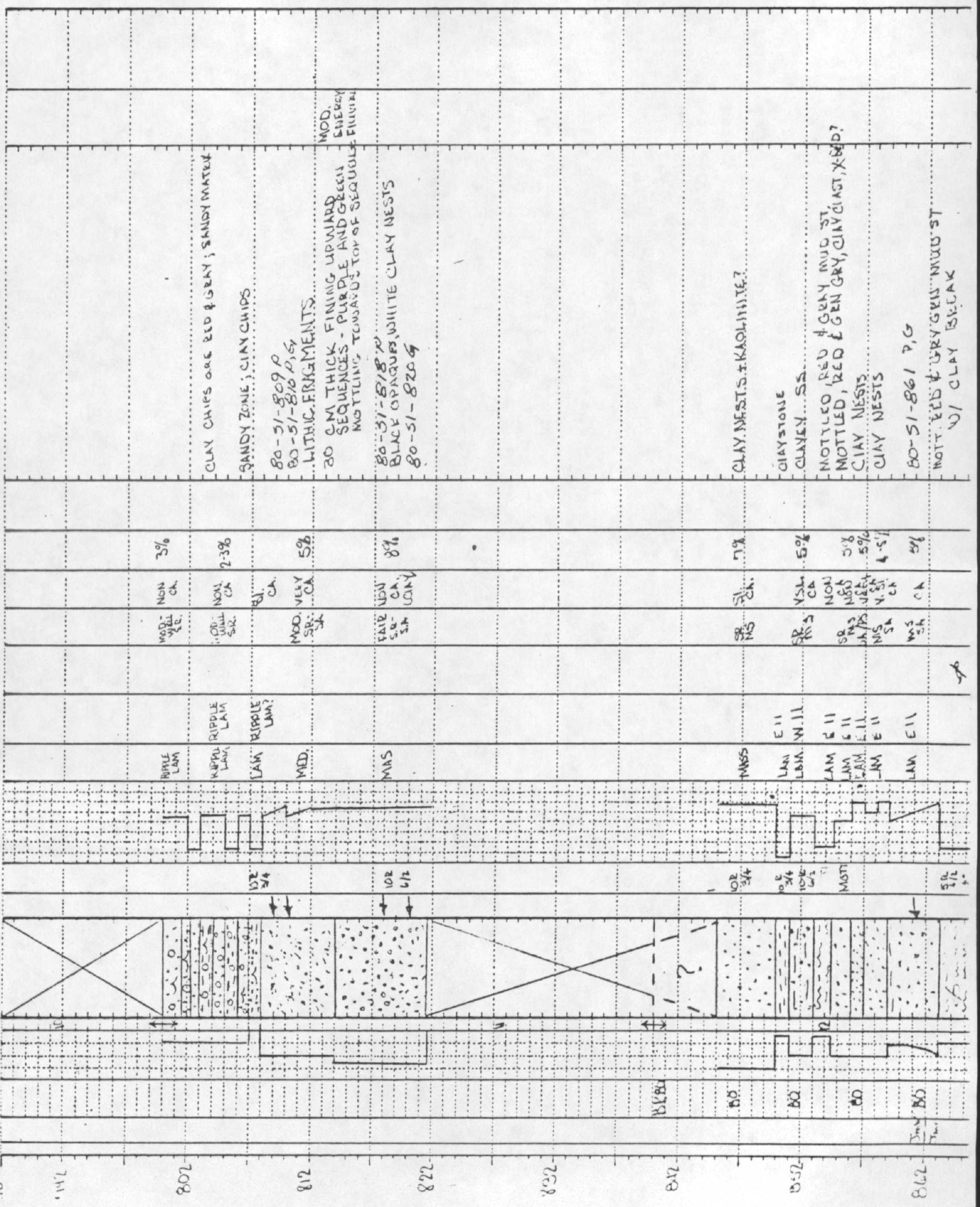

政

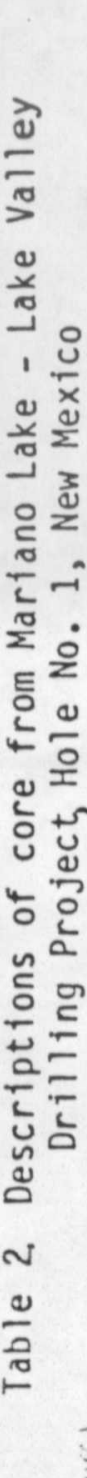


LOCATION....S-1....CORELtO.WE..SeC.....29

T....... $16 . \mathrm{N}$

R......LA..W

STATE ....NEW N.... MEVISQ.

COUNTY MCYINALEY

U.S.G.S. CORE LIBRARY NUMBER

API WELL NUMBER.

\section{的}

竞

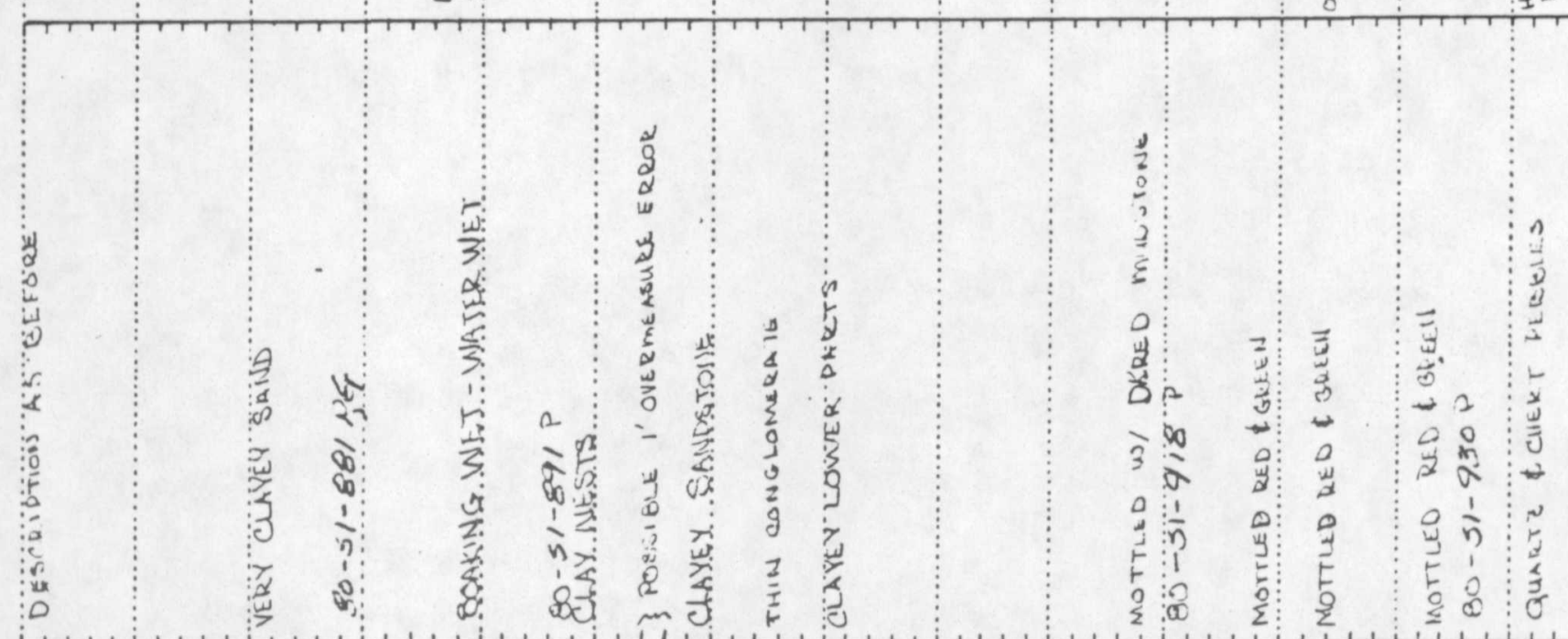

(⿸丆口

म.1.

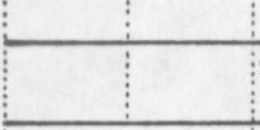

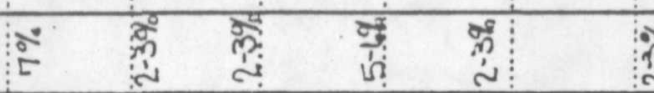

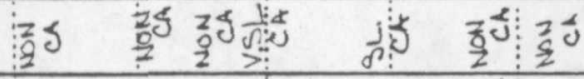

i

\begin{tabular}{|c|c|c|c|c|}
\hline & $\stackrel{d}{r}$ & d & ${ }_{t}^{d}$ & $\stackrel{q}{F}$ \\
\hline & & jư & ju & $i^{-4}$ \\
\hline & & $\frac{1}{3} \frac{1}{3}$ & 30 & $\varepsilon^{\infty}$ \\
\hline
\end{tabular}

$1 \frac{9}{x}$

ख

$\div-$

एँ

E

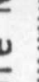

a

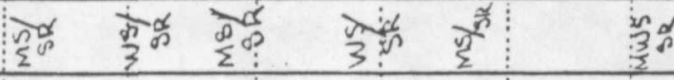

$\frac{3}{3}$

오 운.

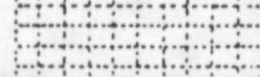

$+\cdots$

ton

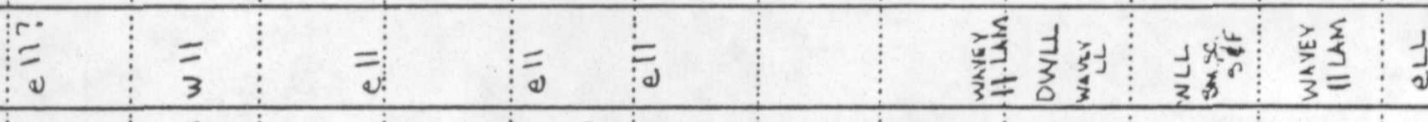

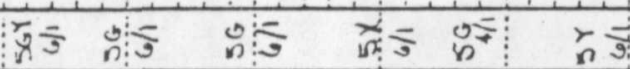

and

$10+\frac{2}{4}$

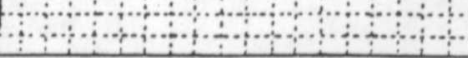

40

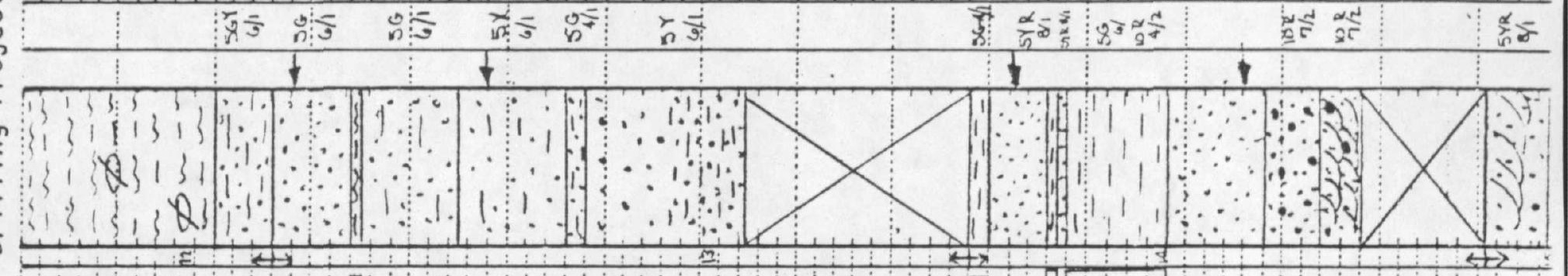

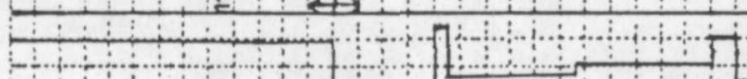

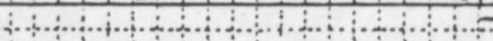

A.

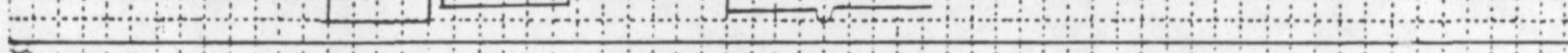

bo

asifieng

is

a

(1)

$\cong$ 


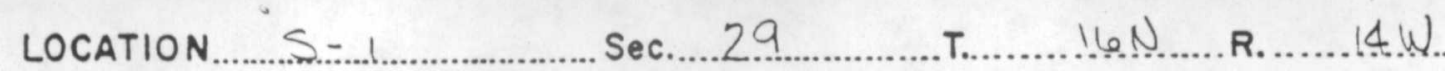

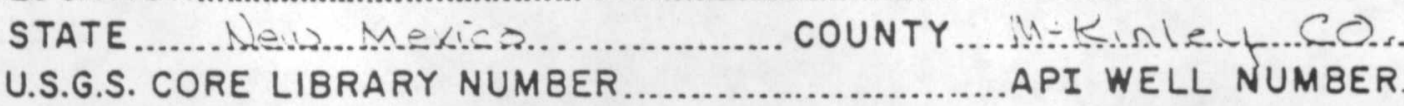

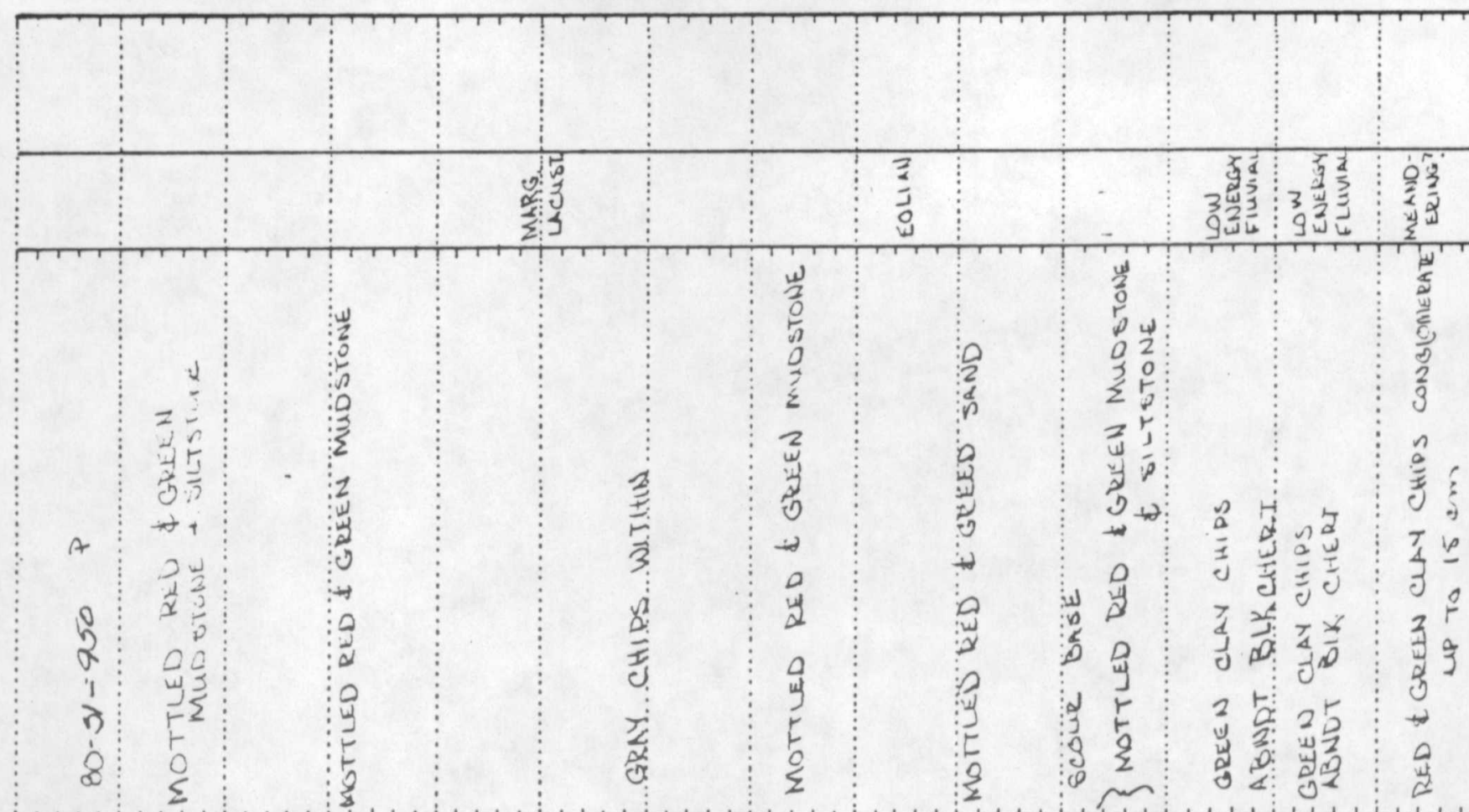

$\frac{1}{2}$

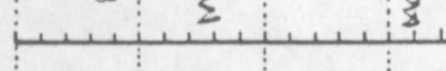

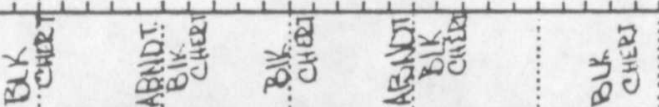
,

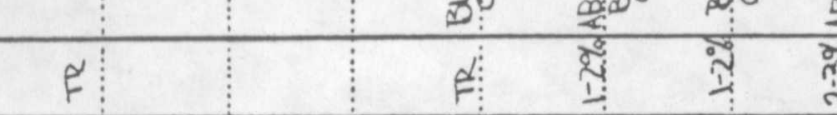

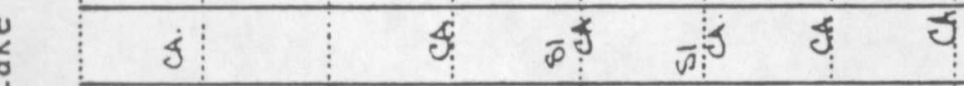
ค:

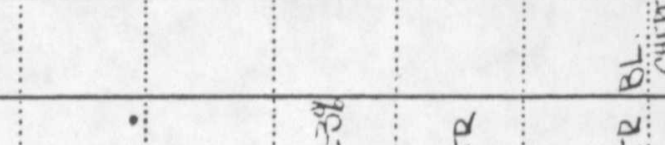
\begin{tabular}{l:c:c}
\hline$y$ & $f$ \\
\hline & $f$
\end{tabular} J $\quad$ 点

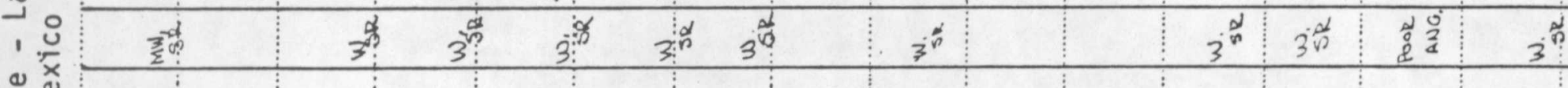
$\frac{\infty}{d} \frac{d}{\Sigma}$

. 幽 오

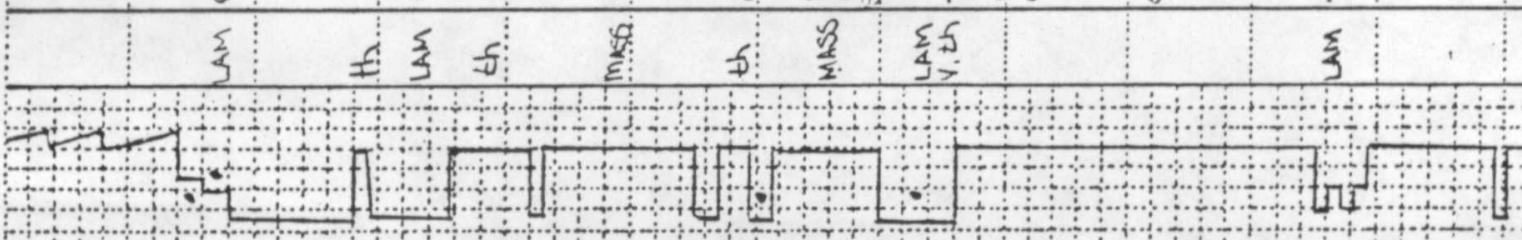

\section{(2)} 1.

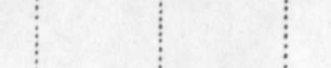

\begin{tabular}{|c|c|c|c|c|c|c|c|c|c|c|c|c|c|}
\hline $\bar{\sigma}$ & $\frac{1}{3}$ & $=$ & $\frac{1}{3}$ & $=$ & $=$ & $\stackrel{\vdots}{d i}$ & 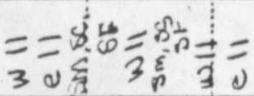 & $\overline{0}$ & 7 & $\bar{\jmath}$ & $\overline{\bar{a}}$ & $\overline{\bar{J}}$ & $\frac{\overline{3}}{3}$ \\
\hline
\end{tabular}

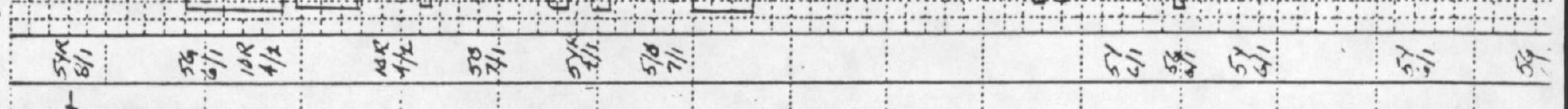

인

望

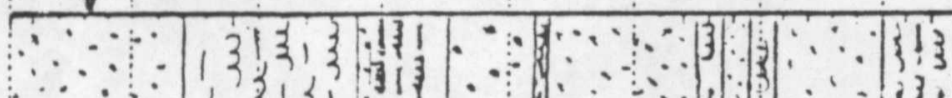

二

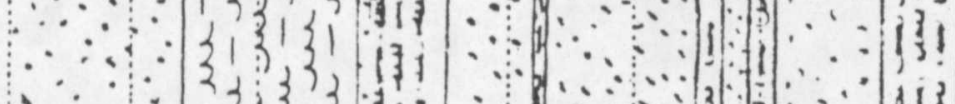

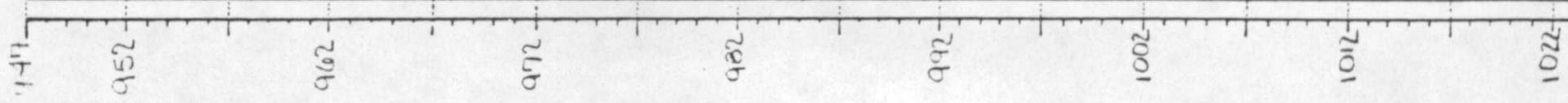




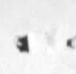

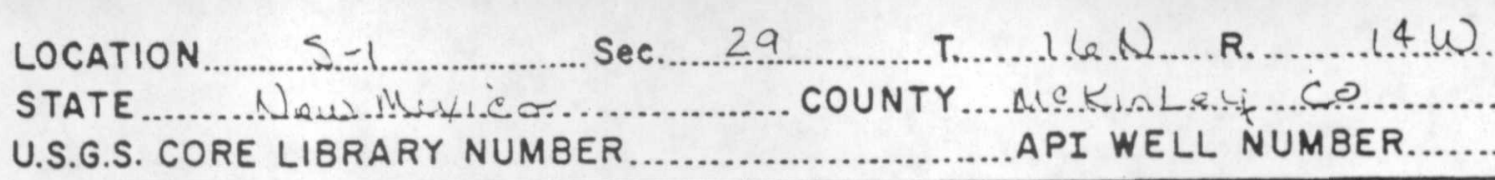

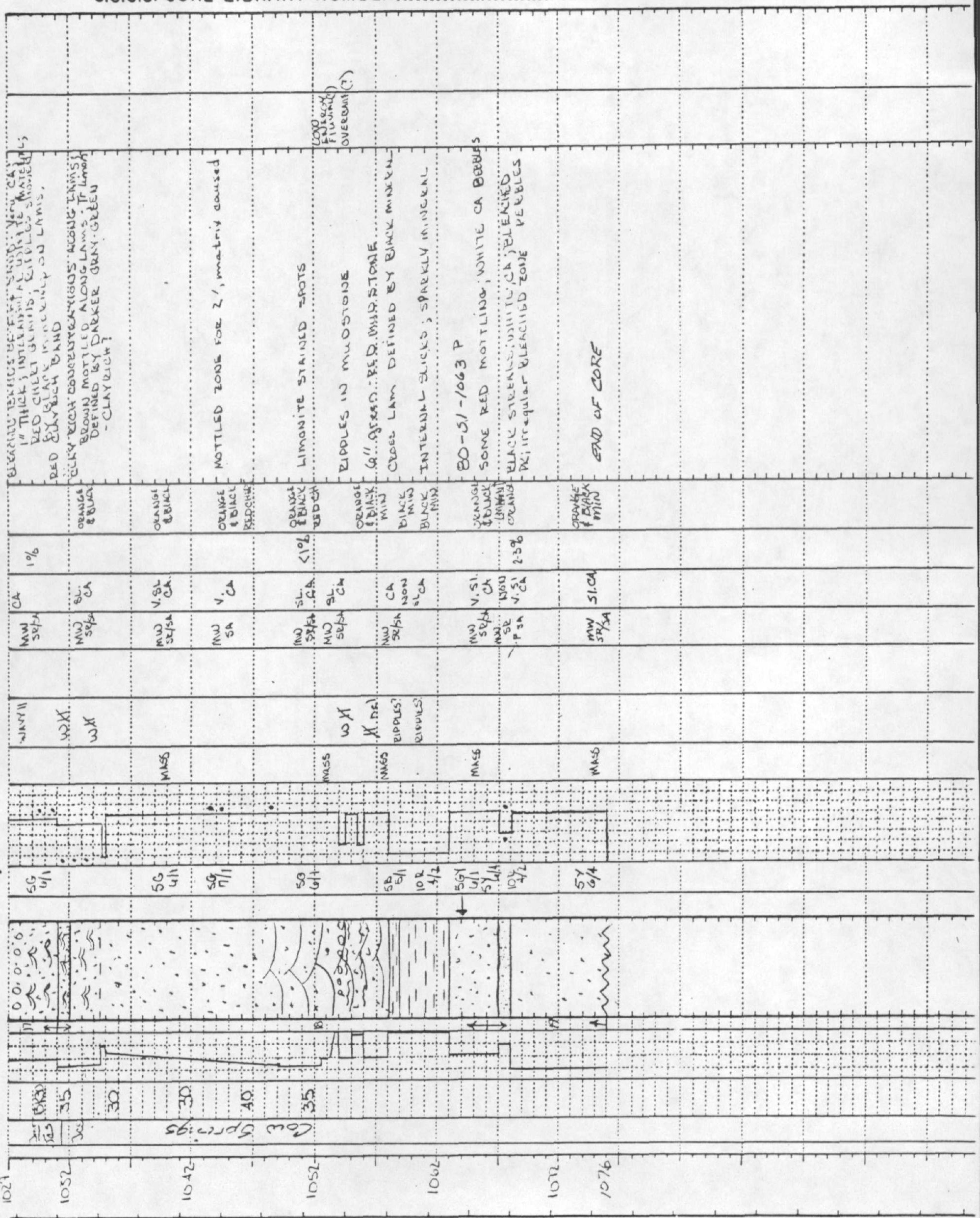

(1) 